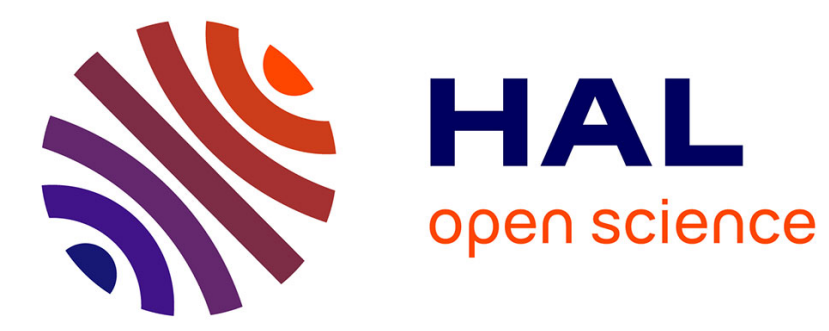

\title{
Local viscosity of a fluid confined in a narrow pore
}

\author{
Hai Hoang, Guillaume Galliero
}

\section{To cite this version:}

Hai Hoang, Guillaume Galliero. Local viscosity of a fluid confined in a narrow pore. Physical Review E : Statistical, Nonlinear, and Soft Matter Physics, 2012, 86, pp.021202. 10.1103/physreve.86.021202 . hal-00778255

\section{HAL Id: hal-00778255 \\ https://hal.science/hal-00778255}

Submitted on 21 Nov 2020

HAL is a multi-disciplinary open access archive for the deposit and dissemination of scientific research documents, whether they are published or not. The documents may come from teaching and research institutions in France or abroad, or from public or private research centers.
L'archive ouverte pluridisciplinaire HAL, est destinée au dépôt et à la diffusion de documents scientifiques de niveau recherche, publiés ou non, émanant des établissements d'enseignement et de recherche français ou étrangers, des laboratoires publics ou privés. 


\title{
Local viscosity of a fluid confined in a narrow pore
}

\author{
Hai Hoang and Guillaume Galliero* \\ Laboratoire des Fluides Complexes et leurs Réservoirs (UMR-5150 with CNRS and TOTAL), Université de Pau et des Pays de l'Adour, \\ Boîte Postale 1155, PAU Cedex 64013, France \\ (Received 9 May 2012; published 20 August 2012)
}

\begin{abstract}
In this paper, molecular dynamics simulations of a simple Lennard-Jones fluid confined in narrow slit pores and undergoing shear have been performed. The aim is to investigate the effects of density inhomogeneities at the fluid-solid interfaces on the shear viscosity profiles. It has been found that the local viscosity was varying strongly with the distance from the solid walls for both dilute and dense fluid states with oscillations correlated to the density ones. To describe the computed viscosity profiles, we propose a scheme that uses the local average density model, combined with an adequate weight function, for the configurational viscosity and a semiempirical model for the translational viscosity. It is shown that the proposed approach is able to provide viscosity profiles in good agreement with those coming from simulations for different pore widths and for different fluid states (dilute to dense).
\end{abstract}

DOI: 10.1103/PhysRevE.86.021202

PACS number(s): 66.20.Cy, 47.11.Mn

\section{INTRODUCTION}

Fluids confined between solid surfaces are generally strongly inhomogeneous in the direction perpendicular to the fluid-solid interfaces because of surface effects (layering of the molecules of the fluid because of adsorption and molecular packing) [1]. This induces local variations in equilibrium and transport properties of the confined fluids [1-4]. Understanding such variations plays an important role in fundamental and applied research for nanofluidics and microfluidics, which has led to an extensive amount of literature on that topic over the past 30 years [1-6].

Classical density functional theory (DFT) has been shown to be able to provide a reliable prediction for strongly inhomogeneous fluids, e.g., density profiles in narrow slit pores [3]. However, the situation is more complex when dealing with the transport properties of inhomogeneous fluids even for simple fluids [6-13]. This is due to the lack of a comprehensive theory to describe the transport properties of nondilute fluids [14] together with difficulties in assessing the results from an experimental point of view. Some attempts exist to deal with that problem. They are mostly based on the Enskog-like kinetic model and molecular dynamics (MD) simulations results and can roughly explain the behavior of the viscosity of inhomogeneous fluids $[8,10,11,15-18]$. However, none of them are able to accurately describe the variation in viscosity of very confined fluids $[10,11,15]$.

Besides the attempts based on the kinetic theory in determining the spatial variation in transport properties of inhomogeneous fluids, there exists another simpler method, which is very easy to implement [6,7]. This method heuristically assumes that a local transport property of an inhomogeneous fluid at a given position is equal to the value of the property at a bulk state corresponding to a locally averaged density around the given position. This approach is called the local average density model (LADM). The LADM reasonably describes the

*guillaume.galliero@univ-pau.fr velocity profiles of fluids confined between solid surfaces and undergoing boundary shears [7,19]. However, the accuracy of such an approach is still questionable as long as an explicit comparison of the local transport property provided by the LADM with the one directly deduced from the MD simulation data does not always exist. Furthermore, previous papers dealing with the LADM were mainly restricted to dense fluids, and it seems that the model cannot be applied to dilute fluids $[6,20]$.

So, this paper aims at improving the description of the local transport properties, limited here to shear viscosity, of strongly inhomogeneous fluid confined in a narrow slit pore. To do so, we employ nonequilibrium molecular dynamics (NEMD) simulations on fluids confined in a narrow pore and undergoing boundary shears as shown in Fig. 1. Then, we determine the local viscosity (separated into translational and configurational contributions) of the confined fluid and evaluate the efficiency of some known models, e.g., the van der Waals (vdW) model and the LADM combined with various weight functions. By doing so, we can directly evaluate the limitations of these models in predicting the local viscosity based on the local thermodynamics properties. Finally, using the results from a previous paper on inhomogeneous fluids without confinement [20], we propose a heuristiclike simple method that can overcome the weaknesses of the classical LADM for low density fluids.

The outline of the paper is as follows: Details on the methodology are presented in Sec. II. Then, the results are provided in Sec. III together with a discussion on the validity of the models aimed at describing the local viscosity of strongly inhomogeneous fluids. Finally, the conclusions are drawn in Sec. IV.

\section{MODEL AND THEORY}

\section{A. Fluid and solid models}

In this paper, we study the shear viscosity of simple fluids confined between solid walls. Interactions between fluid particles and fluid-solid particles are described by a usual 


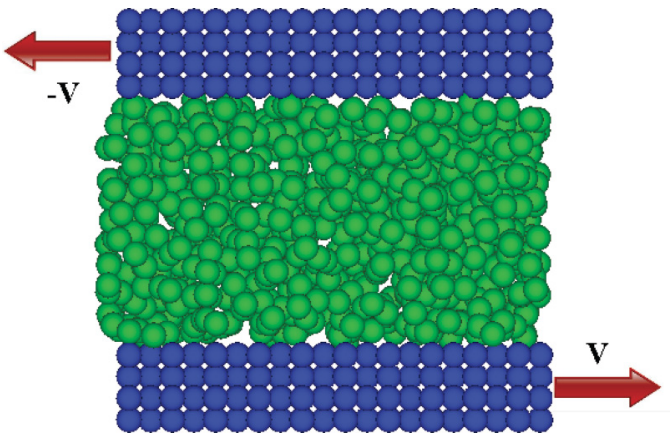

FIG. 1. (Color online) Scheme of the simulation box used in our paper.

truncated Lennard-Jones (LJ) potential [21],

$$
U_{i j}\left(r_{i j}\right)=\left\{\begin{array}{lll}
4 \varepsilon_{i j}\left[\left(\frac{\sigma_{i j}}{r_{i j}}\right)^{12}-\left(\frac{\sigma_{i j}}{r_{i j}}\right)^{6}\right], & \text { if } & r_{i j} \leqslant r_{c}, \\
0, & \text { if } & r_{i j}>r_{c},
\end{array}\right.
$$

where $r_{i j}$ is the distance between particles $i$ and $j, \varepsilon_{i j}$ is the potential depth, $\sigma_{i j}$ is the molecular diameter, and $r_{c}$ is the cutoff radius (taken equal to $2.5 \sigma_{i j}$ in this paper).

The scheme of the simulation box is shown in Fig. 1 in which each wall is made of four atomic layers distributed on a faced centered cubic lattice with the size of the lattice $a=$ $1.6 \sigma_{s s}$, i.e., a number density of the solid wall $\rho_{s} \approx 0.98 / \sigma_{s s}^{3}$. The solid particles are fixed at their sites.

Inhomogeneities of the fluid are induced by interactions between fluid and solid particles. To control these inhomogeneities, the fluid-solid interactions are modulated using a classical $k$ prefactor as

$$
\begin{gathered}
\varepsilon_{s f}=k \varepsilon_{f f}=k \varepsilon, \\
\sigma_{s f}=\sigma_{f f}=\sigma_{s s}=\sigma .
\end{gathered}
$$

By tuning the amplitude of $k$, one can adapt the magnitude of the first adsorbed fluid layer.

In the following, we express the variables in dimensionless units by using the $\mathrm{LJ}$ reduced units. The reduced temperature $T^{*}$, density $\rho^{*}$, stress $P^{*}$, and viscosity $\mu^{*}$ are, thus, defined as Ref. [21]

$$
\begin{aligned}
& T^{*}=\frac{k_{B} T}{\varepsilon}, \quad \rho^{*}=\frac{N_{T} \sigma^{3}}{V}, \\
& P^{*}=\frac{P \sigma^{3}}{\varepsilon}, \quad \mu^{*}=\mu \frac{\sigma^{2}}{\sqrt{m \varepsilon}},
\end{aligned}
$$

where $k_{B}$ is the Boltzmann constant, $N_{T}$ is the total number of atoms contained in the volume $V$, and $m$ is the mass of the fluid particle.

\section{B. NEMD scheme}

The confined fluid is sheared by moving the walls in opposite parallel directions at a constant velocity, see Fig. 1. At the stationary state, this scheme yields a constant shear stress in the fluid phase (this point will be checked in the following). It is important to note that the chosen magnitude of the velocity of the walls should be sufficiently large to obtain a high signal-to-noise ratio but must also satisfy that shear thinning is avoided [20].

\section{Simulation details}

All simulations consist of three steps. First, the confined fluid is equilibrated during a run of $10^{6}$ time steps. Then, the NEMD scheme is applied to shear the confined fluid. Finally, once the steady state is reached, which is ensured by monitoring the evolution of the velocity profile with time, the samplings are performed during 3 to $10 \times 10^{7}$ time steps.

The solid walls are composed of a lattice of $10 \times 10$ unit cells in the $y$ and $z$ directions, respectively, which correspond to a number of wall particles $N_{w}=1600$ and dimensions of the simulated box $L_{y}^{*}=16$ and $L_{z}^{*}=16$. To provide a high signal-to-noise ratio, the walls are moved with a high velocity on the order of 0.5 in reduced units. However, we have verified that the velocities used in the present paper still lead to a linear response without shear thinning (see Sec. III A).

We have used an in-house code to perform the MD simulations. The equations of motion of the particles are solved by employing the Verlet velocity algorithm with a time step $\Delta t^{*}=0.002$. Classical periodic boundary conditions are applied in all directions [21]. To compute interaction force efficiently, we used the Verlet neighbor list [21]. A Berendsen thermostat [22] is applied to the $x$ and $z$ velocity components [23] during the NEMD simulations with the time constant $\tau^{*}=0.2$ and to all three velocity components $(x, y$, and $z$ ) during the equilibrium MD simulations with $\tau^{*}=1$. To compute the local quantities, the simulation box is divided into slabs along the $x$ direction using $\Delta x^{*} \approx 0.11$.

\section{RESULTS AND DISCUSSIONS}

\section{A. Preliminary results}

First, to test the proposed methodology, we have carried out MD simulations on a rather dense fluid confined between solid walls separated by $W^{*}=10$. To generate the initial configuration, we have employed the grand-canonical-like molecular dynamics scheme described in Ref. [24]. In that approach, the pore is simulated in contact with two bulk reservoirs maintained at a given state $\left(T_{\mathrm{Bulk}}\right.$ and $\left.\rho_{\mathrm{Bulk}}\right)$. Then, once the equilibrium is reached, the average density in the center of the pore is extracted and is used to generate the initial configuration of the fluid confined within the slit pore as shown in Fig. 1. The fluid state, employed to perform the preliminary test, corresponds to a bulk fluid at $T^{*}=2$ and $\rho^{*}=0.625$, i.e., a dense supercritical fluid. The $k$ prefactor chosen is equal to 0.387 (moderately adsorbent walls), which provides a maximum of the reduced local density roughly equal to 1 , see Fig. 2(a).

As mentioned previously, during the NEMD simulations, the Berendsen thermostat was employed on the $x$ and $z$ velocity components. So, it is important to check the temperature profile over the $y$ direction (not thermostated) as shown in Fig. 2(b). Two interesting features are observed. First, temperatures in both directions (perpendicular and along the flow) are constant across the entire fluid. Second, the temperature in the $y$ direction is equal to the expected one, even if the thermostat was not applied in this direction. This can simply be understood as a consequence of the equipartition theorem $[21,23]$. 

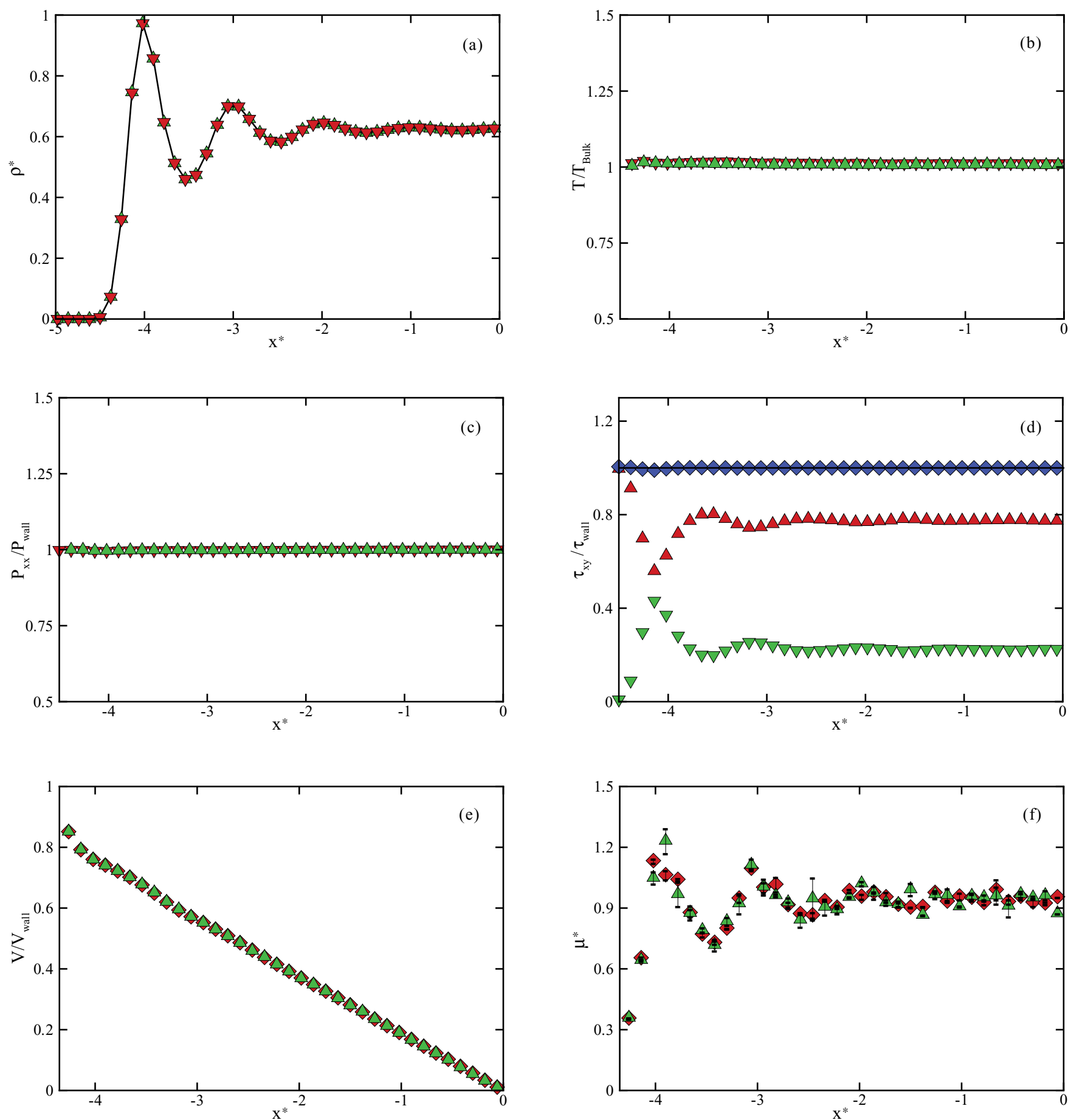

FIG. 2. (Color online) Preliminary results for the state: $\rho_{\text {Bulk }}^{*}=0.625$ and $T^{*}=2$. (a) Density profiles: inverted triangles for equilibrium simulations, triangles for nonequilibrium simulations. (b) Temperature profiles: inverted triangles and triangles for the temperature perpendicular to and along the flow direction respectively. (c) Normal stress profiles: inverted triangles for the normal stress at equilibrium and triangles during nonequilibrium simulations. (d) Shear stress profiles: Diamonds, triangles, and inverted triangles for the total, configurational, and translational shear stresses, respectively. (e) Velocity profiles: diamonds when $V_{\text {wall }}^{*}=0.3$ and triangles when $V_{\text {wall }}^{*}=0.6$. (f) Shear viscosity profiles: diamonds when $V_{\text {wall }}^{*}=0.3$ and triangles when $V_{\text {wall }}^{*}=0.6$.

The momentum conservation equation for a fluid confined in a narrow pore, i.e., at a low Reynolds number and experiencing a boundary shear in the $y$ direction (Couette configuration) should lead to constant shear and normal stresses over the $x$ coordinate $[9,20]$. From the Irving-Kirkwood definition for the pressure tensor, the normal stress $P_{x x}$ and the shear stress $\tau_{x y}$ across a plane parallel with the solid surfaces, i.e., the $y-z$ plane, can be deduced as functions of the $x$ coordinate of the 
plane [9] using

$$
\begin{gathered}
P_{x x}(x)=\frac{1}{V_{s}} \sum_{i} m v_{i, x}^{2} \delta\left(x_{i}-x\right)-\frac{1}{2 A}\left[\sum_{i<j} \frac{x_{i j}^{2}}{r_{i j}} \frac{\partial U_{L J}}{\partial r_{i j}} \frac{1}{\left|x_{i j}\right|}\right. \\
\left.\Theta\left(\frac{x-x_{i}}{x_{i j}}\right) \Theta\left(\frac{x-x_{j}}{x_{i j}}\right)\right], \\
\tau_{x y}(x)=\frac{1}{V_{s}} \sum_{i} m v_{i, x}\left[v_{i, y}-u_{y}(x)\right] \delta\left(x_{i}-x\right) \\
-\frac{1}{2 A}\left[\sum_{i<j} \frac{x_{i j} y_{i j}}{r_{i j}} \frac{\partial U_{L J}}{\partial r_{i j}} \frac{1}{\left|x_{i j}\right|}\right. \\
\left.\Theta\left(\frac{x-x_{i}}{x_{i j}}\right) \Theta\left(\frac{x-x_{j}}{x_{i j}}\right)\right]
\end{gathered}
$$

where $V_{s}$ is the volume of a slab, $A=L_{y} \times L_{z}$ is the area of the $y-z$ plane of the simulation box, $m$ is the mass of a molecule, $v_{i, \alpha}$ is the $\alpha$ component of the velocity of molecule $i, u_{y}(x)$ is the streaming velocity, $x_{i j}$ is the $x$ component of $\mathbf{r}_{i j}, \delta$ is the Kronecker symbol, and $\Theta(x)$ is the Heaviside step function.

To compute the local pressure tensor during the MD simulations, we have employed the volume average method, which is simply a discretization of Eqs. (5) and (6) and is equivalent to the method of planes if a sufficiently fine discretization is employed [25]. So, to divide the simulation box in small slabs perpendicular to the $x$ axis, we have used the width of each slab $\Delta x^{*} \approx 0.11$, which is consistent with what is proposed in Ref. [25] and is sufficient to obtain the normal and shear stress profiles nearly independent of $\Delta x^{*}$. It is worth pointing out that such a formulation of the pressure tensor, Eqs. (5) and (6), implies that the kinetic contributions are assigned to the locations where the particles are, and the configurational contributions are equally distributed between the particles $i$ and $j$ considered. Additionally, we have computed $P_{\text {wall }}$ and $\tau_{\text {wall }}$, which are normal and shear stresses acting on the walls due to intermolecular forces between the fluid and the solid molecules.

Results shown in Figs. 2(c) and 2(d) confirm that the behavior of the confined fluid studied in this paper satisfies the momentum conservation equation. It is worth noting that, even if the density is not constant, see Fig. 2(a), the local shear stress is constant in the whole fluid and is equal to $\tau_{\text {wall }}$.

Equation (6) is composed of two contributions: the first term on the right hand side is the translational contribution, and the second term is the configurational one. Rather surprisingly, the translational contribution increases, and the configurational contribution decreases when the local density increases, see Figs. 2(a) and 2(d). Such a trend is contradictory with what occurs in a homogeneous fluid where the translational contribution decreases with increasing density [26]. However, this can be explained by the fact that, in an inhomogeneous fluid, the number of particles per volume unit increases with density, but, contrary to what occurs in a homogeneous fluid, the mobility of the particles does not decrease $[20,27]$. This can be understood because the momentum transport occurs perpendicular to the dense layer (and the flow) and not parallel to it.
In addition, to test the influence of the shear on the static properties of the confined fluid, we have compared the density and normal pressure profiles at equilibrium with those during nonequilibrium simulations. Results depicted in Figs. 2(a) and 2(c) indicate that, for the shear rate used in the present paper, the shear has a negligible effect on the local density and normal pressure.

It is worth pointing out that, when the strain rate varies rapidly over a length typical of intermolecular correlations, the classical local Newton's law of viscosity must be generalized by a nonlocal constitutive equation [28]. However, in the Couette configuration simulated in this paper, the variation in the gradient of the strain rate is small in the region from the pore center to the first adsorbed layer. This means that the effect of the variation in the strain rate induced from the inhomogeneity of the fluid on the local shear stress can be neglected in this region [29]. It should also be noticed that the remaining region in which the gradient of the strain rate is non-negligible, is rather small, i.e., smaller than $\sigma / 2$. So, one can expect that this effect remains rather limited. To confirm this statement, we have performed NEMD simulations with two different velocities of the solid walls $V_{\text {wall }}^{*}=0.3$ and $V_{\text {wall }}^{*}=0.6$ to compare the results between them.

As shown in Fig. 2(e), the velocity profiles are superposing (when scaled by the velocity of the solid walls) for the two different $V_{\text {wall }}^{*}$ 's tested. This is more obvious when looking at the local viscosity profiles deduced from NEMD simulations and using Newtonian law, i.e., $\mu(x)=\frac{\tau_{x y}(x)}{\left(\frac{d d_{y}(x)}{d x}\right)}$. As shown in Fig. 2(f), they are independent of the velocity of the solid walls. Thus, in the following, a local shear viscosity has been computed using the Newtonian law in which the center finite difference is used to compute the local shear rate, i.e., $d u_{y}(x) / d x$.

It should be mentioned that we have also performed similar checks for a dilute state, i.e., $T^{*}=2$ and $\rho^{*}=0.291$. All the previous findings on the dense state have been confirmed for the dilute state.

\section{B. Viscosity decomposition}

When using Eq. (6) combined with the Newtonian equation, it is possible to separate the viscosity in two contributions, which is crucial to describe the local viscosity of inhomogeneous fluids as we have shown in a previous paper [20]. The first term on the right hand side of Eq. (6) represents the translational contribution, and the second term represents the configurational contribution to the local shear stress [26]. Thus, the local shear viscosity, estimated from the NEMD simulations, can be expressed as the sum of two terms,

$$
\mu(x)=\mu_{t}(x)+\mu_{c}(x),
$$

where $\mu_{t}$ is the local translational shear viscosity computed from

$$
\mu_{t}(x)=\frac{\frac{1}{V_{s}} \sum_{i} m v_{i, x}\left[v_{i, y}-u_{y}(x)\right] \delta\left(x_{i}-x\right)}{\frac{d u_{y}(x)}{d x}},
$$



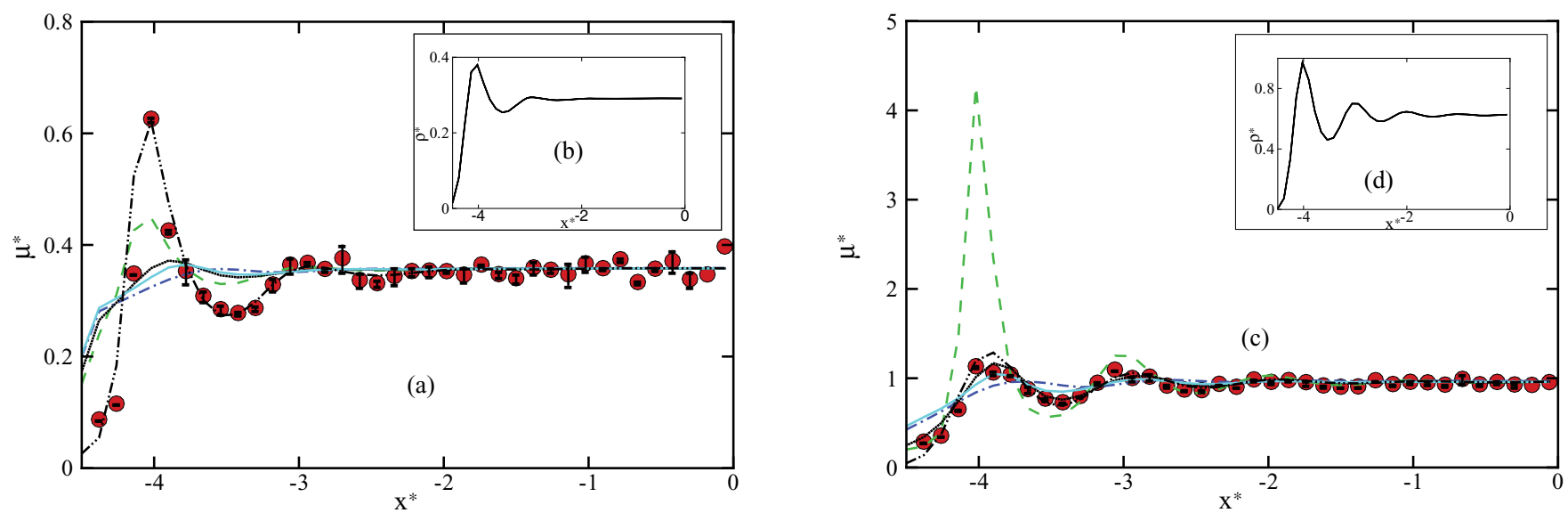

FIG. 3. (Color online) Solid curves in the insets: Shear viscosity profiles with corresponding density profiles. (a) and (b) $T_{\mathrm{Bulk}}^{*}=2.0$ and $\rho_{\mathrm{Bulk}}^{*}=0.291$, (c) and (d) $T_{\text {Bulk }}^{*}=2.0$ and $\rho_{\text {Bulk }}^{*}=0.625$. Circles for the NEMD simulations results, dashed curve (green color) for the vdW model, solid curve (cyan color) for the LADM + F-M model, dotted curve (black color) for the LADM + H-R model, dashed-dotted curve (blue color) for the LADM + Tarazona model, dashed-dotted-dotted curve (black color) for Eq. (22).

and $\mu_{c}$ is the local configurational shear viscosity evaluated thanks to

$$
\mu_{c}(x)=\frac{-\frac{1}{2 A}\left[\sum_{i<j} \frac{x_{i j} y_{i j}}{r_{i j}} \frac{\partial U_{L J}}{\partial r_{i j}} \frac{1}{\left|x_{i j}\right|} \Theta\left(\frac{x-x_{i}}{x_{i j}}\right) \Theta\left(\frac{x-x_{j}}{x_{i j}}\right)\right]}{\frac{d u_{y}(x)}{d x}} .
$$

From a physical point of view, $\mu_{t}$ corresponds to the momentum transfer associated with the displacement (diffusion) of the particles and $\mu_{c}$ corresponds to the contribution due to interaction (collision) between particles. Thus, in a dilute state, $\mu_{t}$ will be the dominant mechanism of momentum transfer, whereas, it will be $\mu_{c}$ for a dense state.

In a homogeneous fluid, the translational viscosity of a LJ fluid is, to a very good extent [26], equal to the "zerodensity" viscosity $\mu_{0}$, deduced from a classical ChapmanEnskog approach [30], i.e.,

$$
\mu_{t}^{*}=\mu_{0}^{*}=\frac{5}{16 \Omega_{v}} \sqrt{\frac{T^{*}}{\pi}},
$$

in which $\Omega_{v}$ is the collision integral.

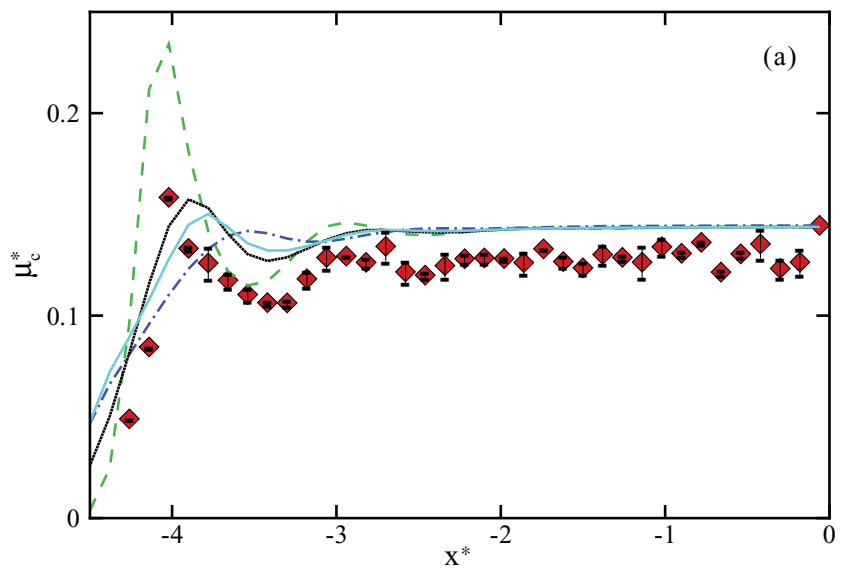

In addition, the configurational viscosity of a LJ homogenous fluid can be described accurately by the correlation developed by Galliero et al. [31] using $T^{*}$ and $\rho^{*}$ as inputs,

$$
\begin{aligned}
\mu_{c, \mathrm{corr}}^{*}\left(T^{*}, \rho^{*}\right)= & \left(e^{b_{2} \rho^{*}}-1\right)+b_{3}\left(e^{b_{4} \rho^{*}}-1\right) \\
& +\frac{b_{5}}{\left(T^{*}\right)^{2}}\left(e^{b_{6} \rho^{*}}-1\right)
\end{aligned}
$$

where the numerical parameters $b_{i}$ have been fitted on extensive MD results. When Eqs. (10) and (11) are combined, the correlation so built yields an estimate of the shear viscosity of a homogenous LJ fluid with a maximum absolute deviation below $5 \%$ compared to MD results for $0 \leqslant \rho^{*} \leqslant 1.275$ and $0.6 \leqslant T^{*} \leqslant 6$.

\section{Local shear viscosity modeling}

So, using the approach described previously, we have computed the viscosity profiles of two different fluids using NEMD simulations, one in a dilute state at $T_{\text {Bulk }}^{*}=2.0$ and $\rho_{\text {Bulk }}^{*}=0.291$ and another one in a dense state at $T_{\text {Bulk }}^{*}=2.0$, $\rho_{\text {Bulk }}^{*}=0.625$, confined in a pore of a width $W^{*}=10$. The

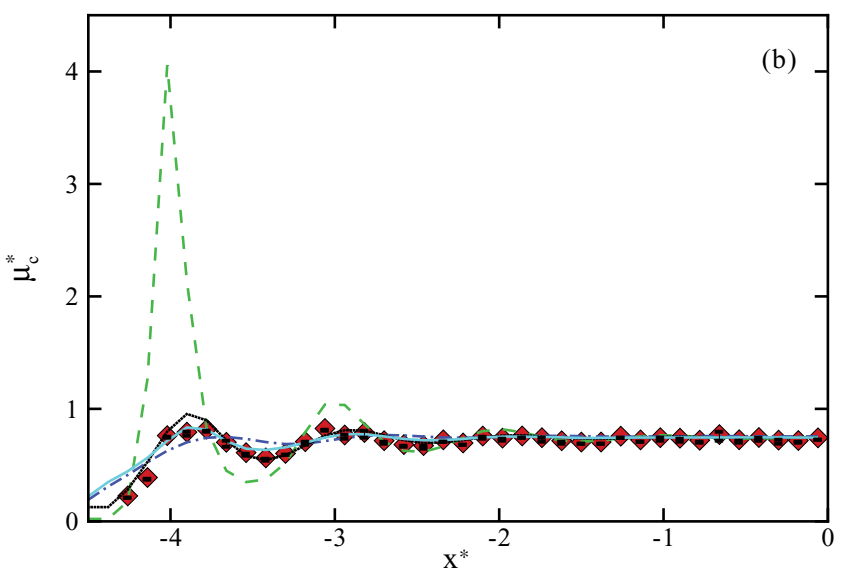

FIG. 4. (Color online) Configurational viscosity profiles. (a) $T_{\text {Bulk }}^{*}=2.0$ and $\rho_{\text {Bulk }}^{*}=0.291$. (b) $T_{\text {Bulk }}^{*}=2.0$ and $\rho_{\text {Bulk }}^{*}=0.625$. The legend is the same as that in Fig. 3. 

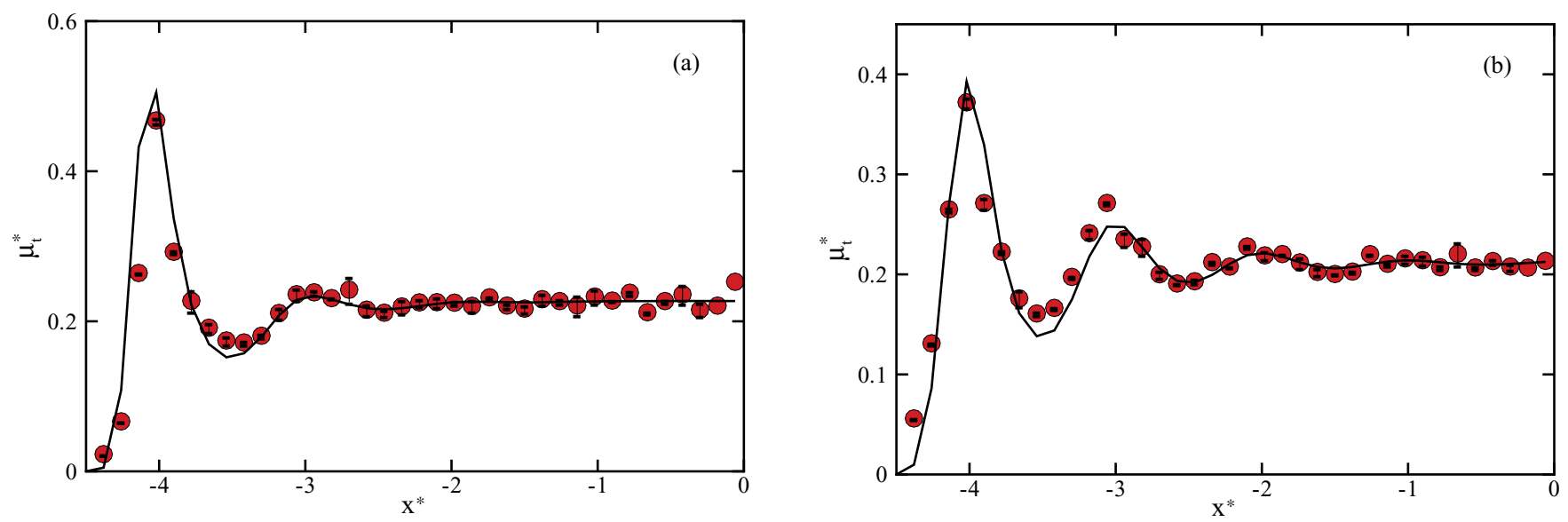

FIG. 5. (Color online) Translational viscosity profiles. (a) $T_{\text {Bulk }}^{*}=2.0$ and $\rho_{\text {Bulk }}^{*}=0.291$. (b) $T_{\text {Bulk }}^{*}=2.0$ and $\rho_{\text {Bulk }}^{*}=0.625$. Circles for NEMD simulations results, solid curves for Eq. (21).

$k$ prefactor (related to adsorption) in Eq. (2) has been taken equal to 0.387 so that the local density remains in the validity range of the LJ shear viscosity correlation, Eq. (11).

The local viscosity in Fig. 3 clearly indicates that the local viscosity deduced from NEMD simulations strongly varies with position (i.e., with density inhomogeneities) for both states. Moreover, the viscosity profiles are strongly correlated to the density ones for the two states studied here, a result which is consistent with previous findings on inhomogeneous systems [20]. To better understand the influence of density inhomogeneities on the local shear viscosity, in the following, we separately consider the effects on the two viscosity contributions.

\section{Configurational viscosity}

By using Eq. (9), we have computed the configurational viscosity profiles for both states during the simulations. As shown in Fig. 4, the configurational viscosity varies with the distance from the solid surface similar to the density profiles. So, as assumed in the DFT approaches for static properties, we have tested to which extent the NEMD configurational viscosity profiles computed using Eq. (9), $\mu_{c}^{*}(x)$, can be

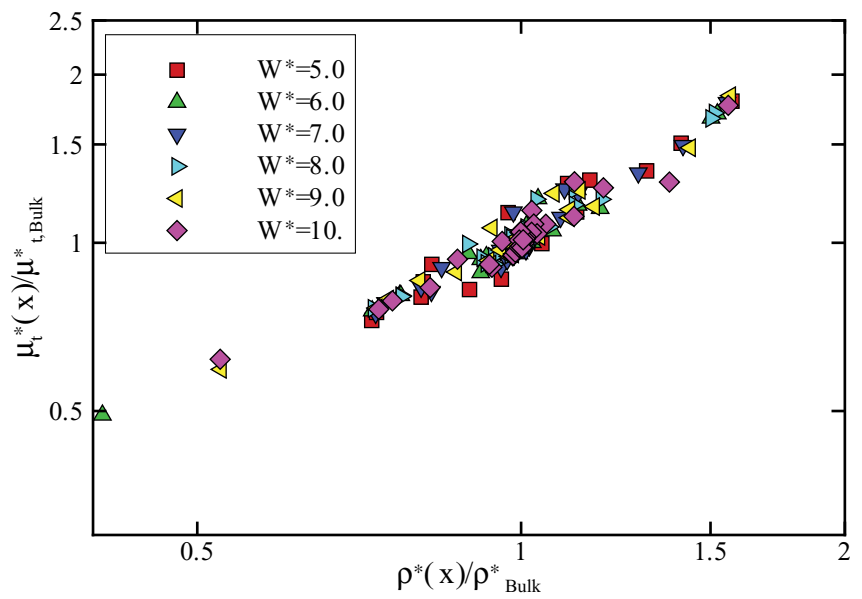

FIG. 6. (Color online) Correlation between $\mu_{t}^{*}(x) / \mu_{t, \mathrm{Bulk}}^{*}$ and $\rho^{*}(x) / \rho_{\text {Bulk }}^{*}$ in the dense state using different pore widths from $5 \sigma$ to $10 \sigma$. deduced from

$$
\mu_{c}^{*}(x)=\mu_{c, \mathrm{corr}}^{*}\left[T^{*}(x), \rho_{\mathrm{eff}}^{*}(x)\right],
$$

where $\mu_{c, \text { corr }}^{*}$ is estimated using Eq. (11) and $\rho_{\text {eff }}^{*}$ is an effective density profile that satisfies the equality in Eq. (12). Thus, the main question is how $\rho_{\text {eff }}^{*}(x)$ should be related to the density profiles obtained during MD simulations, i.e., $\rho^{*}(x)$ ?

To answer that question, we have first tested the simplest approach, called the van der Waals model [20,32], that is defined by

$$
\rho_{\mathrm{eff}}(x)=\rho(x) .
$$

Figure 4 shows that the vdW model leads to a strong overestimation of the variations in $\mu_{c}^{*}$ induced by the density inhomogeneities for both states. This result is consistent with the fact that the configurational viscosity not only is affected by the local thermodynamic properties, but also is affected by the surrounding density, i.e., nonlocal effects are expected. Hence, as the vdW model neglects nonlocal effects, it also fails to yield a good estimate of $\mu_{c}^{*}(x)$.

To include nonlocal effects, we have then used approaches based on the local average density model proposed by Bitsanis et al. [7],

$$
\rho_{\text {eff }}(x)=\int \omega\left(\left|x-x^{\prime}\right|,\{\rho\}\right) \rho\left(x^{\prime}\right) d x^{\prime},
$$

where $\omega(|x|,\{\rho\})$ is a weight function. There exist various forms of the weight function appearing in Eq. (14) that have been proposed to deal with static properties [32,33]. In this paper, we have selected, among them, three well-known different forms. The first one, which is density independent, is the Fischer and Methfessel (F-M) model [33] in which

$$
\omega(|x|)= \begin{cases}\frac{1}{\sigma}, & |x| \leqslant \frac{\sigma}{2} \\ 0, & \text { elsewhere }\end{cases}
$$

The second one, also density independent, the generalized Hard-Rod (H-R) model [33], is defined as

$$
\omega(|x|)= \begin{cases}\frac{6}{\sigma^{3}}\left[\left(\frac{\sigma}{2}\right)^{2}-(|x|)^{2}\right], & \text { when }|x| \leqslant \frac{\sigma}{2}, \\ 0, & \text { elsewhere. }\end{cases}
$$



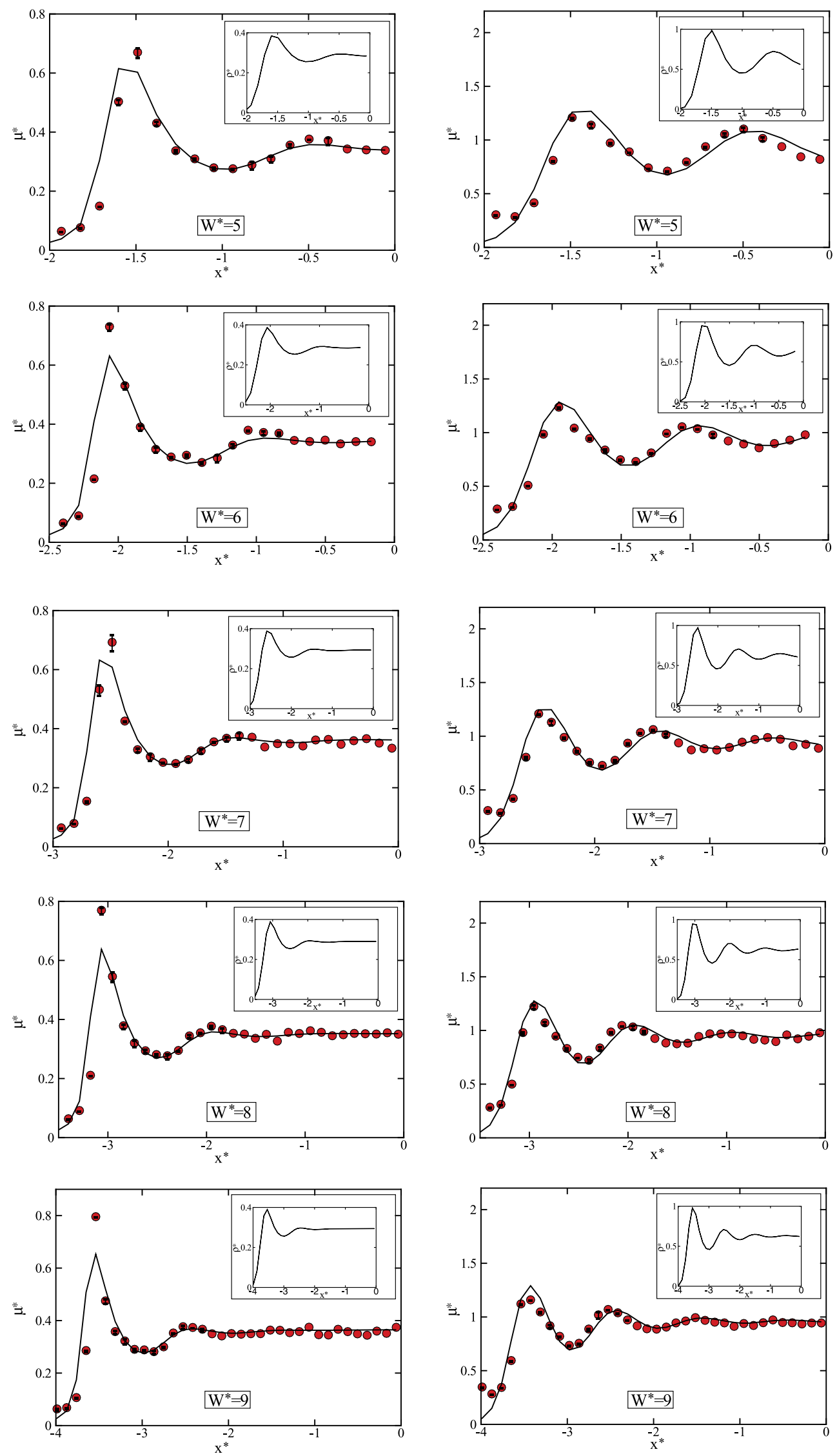

FIG. 7. (Color online) Viscosity profiles for various pore widths (with corresponding density profiles in the insets). Left: dilute state. Right: dense state. Circles for NEMD simulations and curves for Eq. (22). 

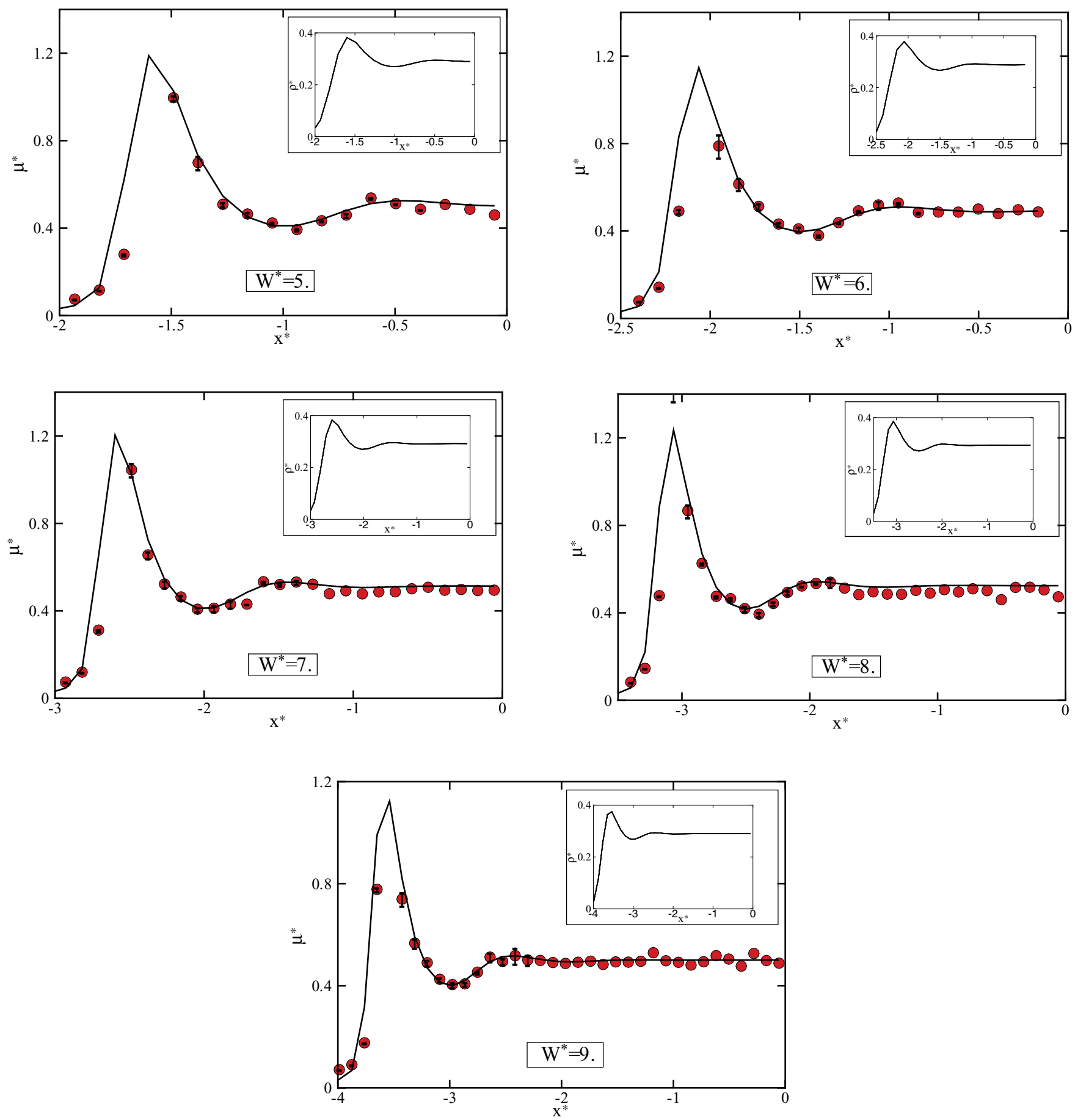

FIG. 8. (Color online) Viscosity profiles for various pore widths for $T_{\mathrm{Bulk}}^{*}=4.0$ and $\rho_{\mathrm{Bulk}}^{*}=0.291$. The legend is the same as that in Fig. 7 .

The last one, which is the most efficient among the three to describe the static properties of confined fluids [33], the Tarazona model, is defined as

$$
\begin{aligned}
\omega\left[\left|x-x^{\prime}\right|, \rho_{\mathrm{eff}}(x)\right]= & \omega_{0}\left(\left|x-x^{\prime}\right|\right)+\omega_{1}\left(\left|x-x^{\prime}\right|\right) \rho_{\mathrm{eff}}(x) \\
& +\omega_{2}\left(\left|x-x^{\prime}\right|\right) \rho_{\mathrm{eff}}(x)^{2}
\end{aligned}
$$

where $\omega_{i=0-2}(|x|)$ are given in Ref. [33]. It is precisely worth the fact that the length, over which the nonlocal effects are taken into account, is equal to $\sigma$ for the first two weight functions, whereas, it is equal to $2 \sigma$ for the last one [32,33].
Figure 4 shows the results obtained when using Eq. (12) combined with Eq. (14) with the three weight functions tested in this paper. From these figures, it is clear that, when nonlocality is introduced using Eq. (14), results can be largely improved compared to the simple vdW model. However, rather surprisingly, the two first weight functions (that introduce nonlocality over a distance equal to $\sigma$ ) yield better results than the Tarazona one (that introduces nonlocality over a distance equal to $2 \sigma$ ), see Fig. 4. This finding is probably related to the fact that thermodynamic quantities are more 
strongly dependent on long-range interactions than transport properties [34,35]. Such an assumption is supported by the fact that hard-sphere approaches are used to estimate viscosity with a reasonable efficiency [14], whereas, such a fluid model is fundamentally inadequate for thermodynamic properties. Another form of support for that assumption comes from the fact that viscosity is only weakly dependent on the cutoff radius [31,36,37], whereas, thermodynamic properties are strongly affected by a small cutoff radius value [21,38].

It should be noted that, among the weight functions with nonlocalities over a distance equal to $\sigma$, the generalized $\mathrm{H}-\mathrm{R}$ function is a more consistent choice because the F-M function, Eq. (15), is strongly discontinuous at $\sigma / 2$, whereas, the H-R is not. Thus, in the following, the H-R weight function is employed.

\section{Translational viscosity}

Similar to what was performed for configurational viscosity, the translational viscosity profiles for both states have been computed during the NEMD simulations. As shown in Fig. 5, the translational viscosity varies appreciably with the distance from the solid surface, i.e., with the densities' inhomogeneities. Such a behavior is contradictory with Eq. (10), which implies a translational viscosity independent of the density. Furthermore, the obvious correlation between the local translational viscosity and the local density is opposite to what is found in homogeneous LJ fluids for which the translational viscosity decreases slightly when the density is increased [26]. This point has already been discussed in Ref. [20] for inhomogeneous fluids without confinement. As explained in this paper, $\mu_{t}^{*}(x)$ is connected to the local number of molecules and the local mobility of each molecule in which the latter is also correlated positively to the local density.

To quantify this correlation between the local translational viscosity and the local thermodynamic conditions, the idea is to quantify the ratio between $\mu_{t}^{*}(x)$ and the translational viscosity of the bulk fluid $\mu_{t \text {, Bulk }}^{*}$, i.e., the translational viscosity of the fluid in the bulk reservoir in contact with the porous medium (at the same chemical potential as the one of the confined fluid). For that purpose, we assume that the translational viscosity is a function of the density and of the insertion probability [20], which leads to

$$
\frac{\mu_{t}(x)}{\mu_{t, \text { Bulk }}}=\frac{g[\rho(x)] f(\rho(x), \exp \{\beta \vartheta[\rho(x), T(x)]\})}{g\left(\rho_{\text {Bulk }}\right) f\left\{\rho_{\text {Bulk }}, \exp \left[\beta \vartheta\left(\rho_{\text {Bulk }}, T_{\text {Bulk }}\right)\right]\right\}},
$$

where $\vartheta$ is the chemical potential, $g$ is a function of the density, and $f$ is a function of the insertion probability. From the thermodynamic equilibrium condition, we can write that

$$
\vartheta[\rho(x), T(x)]=\vartheta\left(\rho_{\mathrm{Bulk}}, T_{\mathrm{Bulk}}\right)
$$

Then, from Eqs. (18) and (19), we can deduce, as a first order approximation, that $\frac{\mu_{t}(x)}{\mu_{t}\left(\rho_{\mathrm{Bulk}}, T_{\mathrm{Bulk}}\right)}$ is a function of $\frac{\rho(x)}{\rho_{\mathrm{Bulk}}}$ only. It should be mentioned that such an approximation is valid only when the variations in $\frac{\rho(x)}{\rho_{\text {Bulk }}}$ are not too large, i.e., when the fluid-solid interactions are not too strong. Starting from that assumption, using the NEMD results obtained, we have found that a simple power law is sufficient to provide reasonable results, see Fig. 6, i.e., the local translational viscosity can be well described by

$$
\mu_{t}(x)=\mu_{t, \text { Bulk }}\left(\frac{\rho(x)}{\rho_{\mathrm{Bulk}}}\right)^{\gamma}
$$

where $\gamma$ is a parameter function of the bulk state $(\gamma \approx 3$ for the dilute state and $\gamma \approx 1.4$ for the dense state). Again, it should be pointed that this relation will remain valid only when the variations in $\frac{\rho(x)}{\rho_{\text {Bulk }}}$ are not too large, i.e., $\frac{\rho(x)}{\rho_{\text {Bulk }}}$ between $2 / 3$ and $3 / 2$.

To estimate the relation between $\gamma$ and $\rho_{\text {Bulk }}$ and $T_{\text {Bulk }}$, we have performed NEMD simulations for 30 different systems keeping $k=0.387$ on a large range of bulk states $\left(T_{\mathrm{Bulk}}^{*}\right.$ varies from 1.5 to 4 and $\rho_{\text {Bulk }}^{*}$ varies from 0.2 to 0.7 ) and various pore widths ( $W^{*}$ varies from 5 to 10 ). Results indicate that $\gamma$ increases with decreasing bulk density and increasing bulk temperature. In other words, the local viscosity is more strongly correlated to $\left(\frac{\rho(x)}{\rho_{\text {Bulk }}}\right)$ at higher bulk temperatures and lower bulk densities. Based on these results, we propose a correlation for $\gamma$,

$$
\gamma=0.8 \frac{\exp \left(0.022 T_{\text {Bulk }}^{2}\right)}{\rho_{\text {Bulk }}} .
$$

As shown in Fig. 5, Eqs. (20) and (21) are able to yield a reasonable $\mu_{t}(x)$ profile based on the local thermodynamic properties.

\section{Local viscosity}

Thus, to quantitatively describe the local viscosity of pure fluids confined in not too adsorbent narrow pores over a large range of thermodynamic conditions, we propose to use

$$
\mu(x) \approx \frac{5}{16 \Omega_{v}} \sqrt{\frac{T(x)}{\pi}}\left(\frac{\rho(x)}{\rho_{\mathrm{Bulk}}}\right)^{\gamma}+\mu_{c, \mathrm{corr}}\left[T(x), \rho_{\mathrm{eff}}(x)\right],
$$

in which $\rho_{\text {eff }}$ is deduced using Eq. (14) with the H-R weight function defined by Eq. (16) and $\gamma$ is obtained by Eq. (21).

As shown in Figs. 3, 7, and 8, the local viscosities predicted from the local thermodynamic condition using Eq. (22) are consistent with the ones provided by NEMD simulations for different thermodynamic conditions (two temperatures and two densities) and various pore widths.

\section{CONCLUSION}

In the present paper, we have investigated the local shear viscosity of Lennard-Jones fluids confined in narrow slit pores by using molecular dynamics simulations of a Couette-like configuration. It is shown that, when using such a configuration with the appropriate parameters, the variation of the gradient of the strain rate is small in the region from the pore center to the first adsorbed layer. The remaining region in which this variation is non-negligible is limited to regions close to the walls that are smaller than $\sigma / 2$. So, it is consistent to define a local viscosity from the classical Newton equation, and a nonlocal constitutive equation is not required in this case.

The local shear viscosity has been found to vary strongly with the distance to the walls and to be dependent on the density inhomogeneities of the fluid. To quantitatively understand 
this behavior, we have decomposed the local viscosity into configurational and translational contributions. Interestingly, it has been found that both contributions vary with the distance to the walls.

NEMD results showed that the local average density model provides a reasonable estimate of the local configurational viscosity when combined with the appropriate weight function, which seems to be the Hard-Rod one for the systems studied here. However, it has been found that this approach is insufficient to yield good results of the viscosity profiles in the dilute state, i.e., when the configurational viscosity is small compared to the translational viscosity.

Based on extensive simulations, we have proposed a simple relation to describe the translational viscosity profiles starting only from the density profiles. Thus, when this relation is combined with the LADM $+\mathrm{H}-\mathrm{R}$ model for the configurational viscosity, we have found that it is possible to quantitatively determine the shear viscosity profiles of a confined LJ fluid for different pore widths (five to ten molecule sizes) and for different fluid states (dilute to dense) when the solid walls are not too adsorbent.

\section{ACKNOWLEDGMENTS}

This work has been supported by the "Failflow" project, which is funded by an advanced grant from the European Research Council. We gratefully acknowledge computational facilities (in link with a "Conseil Regional d'Aquitaine" project) provided by the Pau University and the TREFLE Laboratory (Bordeaux University).
[1] J. Israelachvili, Intermolecular and Surface Forces, Third ed. (Academic, Amsterdam, 2010).

[2] G. Karniadakis, A. Beskok, and N. Aluru, Microflows and Nanoflows (Springer Science + Business Media, New York, 2004).

[3] J. Hansen and I. R. McDonald, Theory of Simple Liquid, Third ed. (Elsevier, London, 2006).

[4] M. Schoen, Computer Simulation of Condensed Phases in Complex Geometries, New Series m: Monographs, Lecture Notes in Physics Vol. m17 (Springer-Verlag, Berlin/Heidelberg, 1993).

[5] K. E. Gubbins and J. D. Moore, Ind. Eng. Chem. Res. 49, 3026 (2010).

[6] S. K. Bhatia, M. R. Bonilla, and D. Nicholson, Phys. Chem. Chem. Phys. 13, 15350 (2011).

[7] I. Bitsanis, T. K Vanderlick, M. Tirell, and H. T. Davis, J. Chem. Phys. 87, 1733 (1987).

[8] L. A. Pohzar and K. E. Gubbins, J. Chem. Phys. 99, 8970 (1993).

[9] B. D. Todd, D. J. Evans, and P. J. Daivis, Phys. Rev. E 52, 1627 (1995).

[10] E. Akhmatskaya, B. D. Todd, P. J. Daivis, D. J. Evans, K. E. Gubbins, and L. A. Pozhar, J. Chem. Phys. 106, 4684 (1997).

[11] X. D. Din and E. E. Michaelides, Phys. Fluids 9, 3915 (1997).

[12] J. Zhang, B. D. Todd, and K. P. Travis, J. Chem. Phys. 121, 10778 (2004).

[13] J. S. Hansen, P. J. Daivis, and B. D. Todd, J. Chem. Phys. 126, 144706 (2007).

[14] J. Millat, J. H. Dymond, C. A. Nieto de Castro, and W. A. Wakeham, Transport Properties of Fluids: Their Correlation, Prediction and Estimation (Cambridge University Press, Cambridge, UK, 2005).

[15] L. A. Pozhar, Phys. Rev. E 61, 1432 (2000).

[16] U. M. B. Marconi and S. Melchionna, J. Chem. Phys. 131, 014105 (2009)

[17] U. M. B. Marconi and S. Melchionna, J. Phys.: Condens. Matter 22, 364110 (2010).

[18] J. Grant Hill, J. Chem. Phys. 135, 044104 (2011).
[19] Z. Guo, T. S. Zhao, and Y. Shi, Phys. Rev. E 71, 035301 (2005).

[20] H. Hoang and G. Galliero, J. Chem. Phys. 136, 124902 (2012).

[21] M. P. Allen and D. J. Tildesley, Computer Simulation of Liquids (Oxford University Press, Oxford, 1989).

[22] H. J. C. Berendsen, J. P. M. Postma, W. F. van Gunsteren, A. Dinola, and J. R. Haak, J. Chem. Phys. 81, 3684 (1984).

[23] D. J. Evans and G. P. Morriss, Statistical Mechanics of Nonequilibrium Liquids (Academic Press, London, 1990).

[24] H. Hoang and G. Galliero, J. Chem. Phys. 136, 184702 (2012).

[25] D. M. Heyes, E. R. Smith, D. Dini, and T. A. Zaki, J. Chem. Phys. 135, 024512 (2011).

[26] G. Galliero and C. Boned, Phys. Rev. E 79, 021201 (2009).

[27] J. Mittal, T. M. Truskett, J. R. Errington, and G. Hummer, Phys. Rev. Lett. 100, 145901 (2008).

[28] B. D. Todd, J. S. Hansen, and P. J. Daivis, Phys. Rev. Lett. 100, 195901 (2008).

[29] B. D. Todd and J. S. Hansen, Phys. Rev. E 78, 051202 (2008).

[30] S. Chapman and T. G. Cowling, The Mathematical Theory of Non-Uniform Gases, Third ed. (Cambridge University Press, Cambridge, UK, 1970).

[31] G. Galliero, C. Boned, and A. Baylaucq, Ind. Eng. Chem. Res. 44, 6963 (2005).

[32] P. Tarazona, J. A. Cuesta, and Y. Martínez-Ratón, Density Functional Theories of Hard Particle Systems, Lecture Notes in Physics Vol. 753 (Springer-Verlag, Berlin/Heidelberg, 2008).

[33] T. K. Vanderlick, L. E. Scriven, and H. T. Davis, J. Chem. Phys. 90, 2422 (1988).

[34] T. Young and H. C. Andersen, J. Phys. Chem. B 109, 2985 (2005).

[35] T. Young and H. C. Andersen, J. Chem. Phys. 118, 3447 (2003).

[36] O.C. Nwobi, L. N. Lon, and M. M. Micci, J. Thermophys. Heat Transfer 12, 322 (1998).

[37] K. Meier, A. Laesecke, and S. Kabelac, J. Chem. Phys. 121, 3671 (2004).

[38] G. Galliero, M. M. Pineiro, B. Mendiboure, C. Miqueu, T. Lafitte, and D. Bessières, J. Chem. Phys. 130, 104704 (2009). 